\title{
Ansiedade e medo em enfermagem médico-cirúrgica
}

\author{
Eduardo Tavares Gomes*, Renata Livia Alves de Sousa Melo**, Eliane Maria Ribeiro de Vasconcelos, D.Sc.***, \\ Eloine Nascimento de Alencar, D.Sc. ${ }^{* * * *}$
}

\begin{abstract}
*Enfermeiro, especialista em Suporte Avançado de Vida pela Faculdade de Enfermagem Nossa Senhora das Graças da Universidade de Pernambuco, aluno do Programa de Especialização em Enfermagem em Cardiologia do Pronto-Socorro Cardiológico Universitário de Pernambuco, **Enfermeira graduada pela Universidade Federal de Pernambuco, aluna do Programa de Especialização em Enfermagem em Cardiologia do Pronto-Socorro Cardiológico Universitário de Pernambuco, ***Enfermeira, Professora adjunto do Departamento de Enfermagem da Universidade Federal de Pernambuco, vice-coordenadora do Programa de Pós-Graduação em Enfermagem - UFPE, ****Professora adjunto do Departamento de Enfermagem da Universidade Federal de Pernambuco e colaboradora do Programa de Pós-Graduação em Enfermagem - UFPE
\end{abstract}

\section{Resumo}

Objetivo: Investigar a produção científica de enfermagem sobre os diagnósticos de enfermagem ansiedade e medo e suas intervençôes na prática assistencial de enfermeiros de clínica médica e cirúrgica. Material e métodos: Trata-se de uma revisão integrativa de literatura, da qual se constituiu um corpus de análise de 16 artigos sobre o tema selecionados nas bases Lilacs e Scielo em janeiro de 2012. Resultados: As categorias temáticas que surgiram foram: ansiedade e medo no período pré-operatório, pós-operatório, antes de exames, diagnósticos e intervençōes para a redução dos níveis de ansiedade. Conclusão: A investigaçâo apontou para o uso de escalas como instrumento para a avaliaçáo de ansiedade e medo pelo enfermeiro e ressaltou a importância da assistência de enfermagem no período pré e pós-operatório.

Palavras-chave: ansiedade, medo, enfermagem médico-cirúrgica, Enfermagem.

\section{Abstract}

\section{Anxiety and fear in medical-surgical nursing}

Objective: This study has investigated the nursing scientific production about the nursing diagnoses of anxiety and fear and their interventions in the care practice of medical-surgical nursing. Method: Integrative literature review by evaluation of 16 articles selected among those published in Lilacs and Scielo databases, in January 2012. Results: The thematic categories that emerged were: anxiety and fear in the preoperative and postoperative periods, before exams, diagnostics, and interventions to reduce anxiety levels. Conclusion: The investigation pointed to the use of scales by nurses as an instrument to assess fear and anxiety and highlighted the importance of nursing care in the preoperative and postoperative periods.

Key-words: anxiety, fear, nursing, medical-surgical nursing, Nursing. 


\section{Resumen}

\section{Ansiedad y miedo en enfermería médico-quirúrgica}

Objetivo: Este estudio investigó la producción científica de enfermería acerca de los diagnósticos de enfermería de ansiedad y miedo y sus intervenciones en la práctica de cuidados de enfermería médico-quirúrgica. Métodos: Se trata de un estudio de revisión integradora de la literatura que evaluó, en enero de 2012, 16 artículos sobre el tema seleccionado en las bases de datos Lilacs y Scielo. Resultados: Las categorías temáticas que surgieron fueron: ansiedad y miedo en el periodo preoperatorio, post-operatorio, antes de los exámenes, y diagnósticos e intervenciones para reducir los niveles de ansiedad. Conclusión: La investigación apuntó el uso de escalas como una herramienta para evaluar el miedo y la ansiedad por enfermeros y destacó la importancia de los cuidados de enfermería en el pre y postoperatorio.

Palabras-clave: ansiedad, miedo, enfermería; enfermería médico-quirúrgica.

\section{Introdução}

Ansiedade e medo são duas condições frequentes nos indivíduos que se submetem aos cuidados de saúde, preenchendo um papel funcional na relação indivíduo-meio ambiente, mas também ocorrendo como sintoma de várias doenças, sob a forma de estresse ou como distúrbio psiquiátrico [1-3].

Alguns autores definem ansiedade como um sentimento vago e desagradável, um mal-estar psíquico e somático, relacionando-a ao medo e à apreensão, a qual é caracterizada por tensão ou desconforto derivado de antecipação de perigo, de algo desconhecido ou estranho [3]. A ansiedade é também entendida como uma resposta fisiológica do organismo desencadeada por eventos estressantes, porém necessária para o indivíduo enfrentar as situaçóes de risco [4-5]. Acredita-se que reaçóes exageradas ao estímulo ansiogênico se desenvolvem, mais comumente, em indivíduos com uma predisposição neurobiológica herdada [3].

Ansiedade e medo passam a ser reconhecidos como patológicos quando são exagerados, desproporcionais em relaçáo ao estímulo, ou qualitativamente diversos do que se observa como norma em determinado grupo e causam interferência perniciosa na qualidade de vida, com prejuízo do conforto emocional ou do desempenho diário do indivíduo, com longa duração. A maneira prática de se diferenciar ansiedade normal de ansiedade patológica é basicamente avaliar se a reaçáo ansiosa é de curta duração, autolimitada e relacionada ao estímulo do momento ou não [3]. O traço de ansiedade é uma tendência moldada pela personalidade de desenvolver mais ou menos ansiedade, diante das experiências de vida, ou seja, é uma capacidade de resposta com a propriedade de modificar o estado de ansiedade [4].

A associação entre ansiedade e medo é frequente em diversos estudos e muitos pacientes com altos escores de medo também apresentam altos escores de ansiedade ou não apresentam diferenças significativas do uso dos diagnósticos. Esta forte correlação constitui uma justificativa para alguns autores defenderem a existência de uma síndrome resultante do conjunto de manifestaçóes de ambos os diagnósticos [6-7]. A diferenciação entre medo e ansiedade está, principalmente, na diferenciação pelo paciente do objeto causador do sentimento [8].

Ansiedade e medo são Diagnósticos de Enfermagem (DE) presentes nas primeiras propostas de taxonomia. São diagnósticos utilizados de forma corriqueira não apenas nas unidades de saúde que passaram pelo processo de Sistematização da Assistência de Enfermagem, mas também constante nos registros e anotaçóes realizados pelos profissionais de enfermagem. Enfermeiros e técnicos registram usualmente os termos "ansioso" e "com medo" nos prontuários de seus pacientes, de forma empírica e baseado principalmente no relato verbal [9].

O presente estudo verificou na literatura atualidades que possam contribuir para o uso seguro dos $\mathrm{DE}$ ansiedade e medo e suas intervençôes na prática assistencial em clínica médica e cirúrgica.

\section{Material e métodos}

Trata-se de uma revisão integrativa sobre o tema. Este é um dos métodos utilizados na prática baseada em evidências, aceita por organizaçóes inter- 
nacionais de pesquisa, náo só em Enfermagem, mas em toda área da saúde, por permitir a incorporação das evidências na prática clínica. Inclui a análise e síntese de pesquisas relevantes para o apoio à prática clínica e a tomada de decisão [10-11]. Por propor-se a oferecer o conhecimento atual da literatura sobre determinado tema, a revisão integrativa tem exercido papel fundamental para o aprimoramento dos Diagnósticos de Enfermagem, como etapa inicial do processo de validação [12].

Pode-se enumerar em seis fases o processo de elaboração de uma revisão integrativa $[10,11,13]$ : $1^{\text {a }}$ Fase: Identificação do tema ou questionamento da revisão integrativa elaboração da pergunta norteadora; 2a Fase: Amostragem ou busca na literatura; $3^{\text {a }}$ Fase: Coleta de dados - categorizaçáo dos estudos; 4a Fase: Análise crítica dos estudos incluídos na revisão integrativa; 5a Fase: Interpretação dos resultados; 6a Fase: Síntese do conhecimento evidenciado nos artigos analisados ou apresentação da revisão integrativa.

A questão norteadora escolhida foi: quais os conhecimentos e reflexóes da enfermagem sobre ansiedade e medo em pacientes de Clínica Médica e Cirúrgica?

O período de coleta de dados compreendeu o mês de janeiro de 2012. Foram utilizados os descritores ansiedade, medo e enfermagem e seus cruzamentos, para realização de busca nas bases de dados Scielo e BDENF. Compuseram o corpus de análise artigos em português, publicados em periódicos de enfermagem, com no mínimo um autor enfermeiro, estudando ansiedade e medo em Clínica Médica e Cirúrgica. A busca criteriosa nas bases de dado revelou os 16 artigos que elencaram a amostra.

\section{Resultados}

A maioria dos estudos foi realizada na última década $(75,1 \%)$. O desenho de estudo descritivo-exploratório foi o mais utilizado (50\%). Metade dos estudos utilizava questionários próprios ou entrevistas para avaliaçáo do medo e ansiedade através de características clínicas ou avaliação cognitiva. (Tabela I)

O questionário autoaplicável conhecido como Inventário de Ansiedade Traço-Estado (IDATE) foi utilizado em sete estudos. Esse inventário é composto por duas escalas distintas para medir conceitos subjetivos: ansiedade-traço e ansiedade-estado. Ansiedade-traço pode ser definida como a propensão à ansiedade, permanente no indivíduo. A ansiedade-estado seria momentânea, conscientemente percebida, eventual, modificando-se com o tempo e sendo influenciada por fatores externos. Essas escalas constam de 20 afirmaçóes, em que o sujeito indica a intensidade naquele momento (estado) ou a frequência com que ocorre (traço), através de uma escala de quatro pontos ( $1 \mathrm{a} 4)$. Os escores mais próximos de 80 , pontuação máxima, indicam maior nível de ansiedade [14].

Tabela I - Distribuição dos artigos segundo o ano de publicação, tipo de estudo, instrumento de coleta. Recife/PE, 2012.

\begin{tabular}{llc}
\hline \multicolumn{1}{c}{ Variável } & $f$ & $\%$ \\
\hline Ano & & \\
\hline $1998-2000$ & 4 & 25,0 \\
$2000-2005$ & 5 & 31,3 \\
$2005-2011$ & 7 & 43,8 \\
\hline Tipo de estudo & & \\
\hline Descritivo-exploratório & 8 & 50,0 \\
Experimental & 2 & 12,5 \\
Ensaio Clínico & 1 & 6,3 \\
Estudo de Caso & 2 & 12,5 \\
Qualitativo & 2 & 12,5 \\
Reflexão & 1 & 6,3 \\
\hline Instrumento de coleta & & \\
\hline IDATE* & 7 & 35,0 \\
HAD** & 1 & 5,0 \\
Inventário de estratégias de & 1 & 5,0 \\
coping & & \\
Entrevista & 5 & 25,0 \\
Questionário próprio & 5 & 25,0 \\
Não se aplica & 1 & 5,0 \\
\hline
\end{tabular}

*Inventário State-Trate Anxiety; ${ }^{* *}$ Escala Hospitalar de Ansiedade e Depressão.

A Escala Hospitalar de Ansiedade e Depressão avalia aspectos cognitivos apenas, sem avaliar sinais e sintomas físicos, por isso, é muito utilizada em doenças físicas [15-17]. É composta por duas subescalas, para ansiedade e depressão, com sete itens cada e pontuaçáo de 0 a 21 e foi traduzida e validada para o português por especialistas [17].

\section{Discussão}

\section{Ansiedade e medo no período pré-operatório}

Muitos dos pacientes admitidos em uma instituição hospitalar para fins terapêuticos apresentam-se sem informaçóes e orientaçóes necessárias 
sobre as intervençóes, carregando mitos e ideias do imaginário que refletem no trâmite a que se submetem com reaçóes negativas. A experiência de uma intervenção como, por exemplo, o procedimento cirúrgico-anestésico é um processo que remete a "aproximar-se da morte", principalmente quando há inconsciência causada pelos fármacos, gerando comumente a ansiedade e o medo [14,18-19].

Em estudo do tipo descritivo-exploratório, através de entrevistas com 13 pacientes no período pré-operatório de cirurgia cardíaca identificou-se em $100 \%$ dos entrevistados o Diagnóstico de Enfermagem Medo [20]. Outros autores encontraram resultados semelhantes, através de um mesmo desenho de estudo, utilizando-se de questionário próprio aplicado a 50 pacientes que seriam submetidas à cirurgia ginecológica. Os diagnósticos Ansiedade e Medo estavam presentes em $96 \%$ das pacientes e a Síndrome da Ansiedade Perioperatório atingiu o mesmo [7]. Uma série de estudos de 20 casos de pacientes no período pré-operatórios, realizados através de entrevista semiestruturada, identificou duas categorias de sentimentos, os de apreensão (medo, ansiedade, preocupação, receio, cisma e nervosismo) e sentimentos positivos e de esperança [21]. Por fim, outros autores avaliaram que as estratégias de enfrentamento no período pré-operatório interferiam nos níveis de ansiedade [4].

\section{Ansiedade no período pós-operatório}

O evento cirúrgico pode suscitar, como visto, ansiedade e medo no período pré-operatório. Em alguns pacientes a ansiedade pode permanecer no período pós-operatório em virtude da expectativa das mudanças advindas com a intervenção em sua rotina.

Em um estudo de caso de uma paciente no período pós-operatório de uma cirurgia cardíaca de emergência, foi verificado que a ansiedade estava presente no período e os autores sugeriram associação a falta de tempo para o preparo prévio adequado do paciente [22]. Outro estudo, com objetivo de verificar a correlaçáo entre o traço e o estado de ansiedade no período pré-operatório e a influência da ansiedade na resposta do paciente no período pós-operatório imediato, em sala de recuperação pós-anestésica, avaliou através do IDATE 33 pacientes cirúrgicos, encontraram correlação positiva no período pré-operatório entre ansiedade traço e estado, mas não foi significativa a correlação imediatamente após a cirurgia [14]. Deve ser considerado, nesse caso, o tipo de cirurgia, anestésico e preparo prévio, de ordem psicoemocional.

\section{Ansiedade perante exames e diagnósticos}

Não apenas o procedimento cirúrgico é fonte de ansiedade e medo no ambiente hospitalar, mas também procedimentos de enfermagem, clínica médica, realizaçáo de exames e diagnósticos. Várias são as expectativas e conteúdos emocionais envolvidos no ato de "esperar" por algum acontecimento ou por alguém. Em situações de hospitalização, tratamentos médicos e exames diagnósticos, o período de espera pode tornar-se angustiante, podendo levar ao stress e à ansiedade. Estudos correlacionam a espera para um exame diagnóstico invasivo a ansiedade relacionada à ameaça da integridade física e ao bem-estar, como também às implicaçóes advindas do resultado diagnóstico [23]. Os sentimentos envolvidos na descoberta do diagnóstico são as preocupaçóes relacionadas aos internamentos, aos procedimentos, às mudanças nos hábitos e, principalmente, ao medo da morte [21,22-25]. Com a acomodação das ideias e estratégias de enfrentamento, os sentimentos de apreensão mudam para um conteúdo mais esperançoso entre o momento do diagnóstico até o momento da cirurgia [21].

Em estudo ocorrido em uma clínica radiológica, avaliou-se que a ansiedade e a claustrofobia impediram a realizaçáo de 3\% dos exames de ressonância magnética acompanhados pelos pesquisadores [25]. Os altos níveis de ansiedade são uma condição que leva o indivíduo a diminuir sua capacidade de atenção e de resposta aos estímulos externos, com fixação na causa tensora, impedindo nos momentos mais agudos a realizaçáo de procedimentos [2]. Outro trabalho da amostra, ao se propor identificar o nível de ansiedade de acompanhantes e clientes submetidos à cineangiocoronariografia pela primeira vez, no período de espera do exame, encontrou ainda que o nível de ansiedade dos acompanhantes era médio, enquanto o dos pacientes era baixo, indicando a necessidade do olhar à família como fator predisponente e agravante da ansiedade e do medo [22].

\section{Intervençóes para reduçáo dos níveis de ansiedade}

Enfrentamento pode ser entendido como o conjunto de esforços desenvolvidos para manejar ou lidar com as solicitaçóes externas e internas, 
que são avaliadas como excessivas ou acima de suas possibilidades. É um esforço para dominar, tolerar ou reduzir um conflito entre novas demandas, é uma ação dirigida para a resolução de algum problema, dependente de avaliação e de que recursos que o indivíduo dispóe para enfrentar a situação [18].

A enfermagem tem papel importante em reconhecer e fortalecer as estratégias de enfrentamento do medo e ansiedade relacionando-os aos estressores do ambiente hospitalar. Um estudo que compôs a amostra encontrou resultados que mostram os benefícios da visita pré-operatória de enfermagem correlacionando-a a diminuiçáo do nível de ansiedade dos pacientes. Houve redução da media dos escores de ansiedade no grupo com visita quando comparado ao grupo controle, bem como no número de pessoas com níveis elevados e moderados de ansiedade [27]. Outro estudo, avaliando 146 pacientes submetidos à revascularização miocárdica, constatou que as pessoas acompanhadas de acordo com o protocolo de consultas de enfermagem tiveram menor percentual de ansiedade e depressão, após seis meses de acompanhamento [15].

A visita de enfermagem pré-operatória deve ser instrutiva acerca do procedimento e do pós-operatório e atenta para identificar características definidoras de medo e ansiedade e correlacionar os diagnósticos de enfermagem como potenciais dificultadores. Além das orientaçóes individualizadas nas visitas, os enfermeiros podem criar um espaço para troca de vivências e para discussáo, como os grupos terapêuticos [28].

Das diversas linhas de terapias integrativas e complementares, as quais são da competência dos enfermeiros, ressurgiu a utilizaçáo das máos como uma ferramenta eficiente. Várias são as técnicas utilizadas, abrangendo desde a pressão digitálica até o toque terapêutico, tendo nas massagens as mais conhecidas representantes dessa categoria [5,29]. A calatonia, estudada em um dos trabalhos do corpus de análise, é uma técnica de relaxamento composta de toques sutis em nove pontos do corpo, demorando-se de um a três minutos em cada ponto. São considerados pontos: regióes ungueais de todos os artelhos (dedos dos pés), as plantas dos pés, os tornozelos, as convergências tendinosas do tríceps, das regióes posteriores das pernas (panturrilhas) e a região posterior do pescoço. O processo é realizado em silêncio, ocorrendo apenas o toque das mãos e dos dedos sem pressão ou fricção nos pontos referidos [5].
As estratégias de educação do paciente favorecem também ao enfrentamento. Autores sugerem além das orientaçôes verbais aos pacientes em visita pré-operatória, a construção de manuais e materiais educativos para pacientes e acompanhantes, visando diminuir os níveis de ansiedade [25-26]

\section{Conclusão}

A cirurgia e a anestesia, bem como os demais procedimentos diagnósticos e de enfermagem são situaçóes impostas pela necessidade, exigindo um enfrentamento e favorecendo sentimentos diversos, comumente a ansiedade e o medo.

Verificou-se a importância do papel do enfermeiro atuando junto ao paciente antes dos procedimentos que precipitam a ansiedade e o medo, desenvolvendo açóes educativas e intervençôes que visem o relaxamento e a diminuição dos níveis de ansiedade. A criação de grupos para discussão e preparação pré-operatória pode ser realizada por enfermeiros, considerando-os habilitados na condição de educadores em saúde e participantes dos processos de cuidar relacionados ao ato cirúrgico.

A investigação revelou que os enfermeiros que pesquisaram o tema fizeram uso de escala validadas e difundidas nas clínicas médica e cirúrgica, mas fizeram pouco uso das taxonomias de enfermagem. Sugere-se, dessa forma, a validaçáo do uso de escalas como características definidoras dos Diagnósticos de Enfermagem Ansiedade e Medo.

\section{Referências}

1. Loreto G. Conceptual dimension of anxiety. Neurobiologia 1982;45(1):3-12.

2. Dractu L, Lader M. Anxiety - Concept, classification and biology: a contemporany interpretation of the literature. J Bras Psiquiatr 1993;42(1):19-32.

3. Castillo ARGL, Recondo R, Asbahr FR, Manfro GG. Transtornos de ansiedade. Rev Bras Psiquiatr 2000;22:20-3.

4. Medeiros VCC, Peniche ACG. A influência da ansiedade nas estratégias de enfrentamento utilizadas no período pré-operatório. Rev Esc Enferm USP [online] 2006;40(1):86-92.

5. Nosow V, Peniche ACG. Paciente cirúrgico ambulatorial: calatonia e ansiedade. Acta Paul Enferm 2007;20(2):161-7.

6. Suriano MLF. Comportamento das características definidoras dos diagnósticos de enfermagem medo e ansiedade identificados no pré-operatório imediato de pacientes submetidos a cirurgias eletivas [tese]. São Paulo: Universidade Federal de São Paulo. Escola Paulista de Medicina; 2005. 
7. Suriano MLF, Lopes DCF, Macedo GPOS, Michel JLM, Barros ALBL. Identificação das características definidoras de medo e ansiedade em pacientes programadas para cirurgia ginecológica. Acta Paul Enferm 2009;22:928-34.

8. Carpenito-Moyet LJ. Manual de Diagnósticos de Enfermagem. 11a ed. Porto Alegre: Artmed; 2008.

9. Gomes ET, Melo RLAS, Vasconcelos EMR, Alencar EM. Uso dos diagnósticos de Enfermagem ansiedade e medo nas clínicas médica e cirúrgica de um hospital universitário. Rev Pesq: Cuid Fundam 2012;4(2):241926.

10. Mendes KDS, Silveira RCCP, Galvão CM. Revisão integrativa: método de pesquisa para a incorporação de evidências na saúde e na enfermagem. Texto Contexto Enferm 2008;17(4):758-64.

11. Aguiar ESS, Gomes IP, Fernandes MGM, Silva AO. Representaçóes sociais do cuidar de idosos para cuidadores: Revisão Integrativa. Rev Enferm UERJ 2011;19(3):485-90.

12. Pompeo DA, Rossi L.A, Galvão CM. Revisão integrativa: etapa inicial do processo de validaçáo de diagnóstico de enfermagem. Acta Paul Enferm 2009;22(4):434-8.

13. Polit DF, Beck CT, Hungler BP. Fundamentos de pesquisa em enfermagem: métodos, avaliação e utilização. Porto Alegre: Artmed; 2004.

14. Peniche ACG, Jouclas VMG, Chaves ECA. A influência da ansiedade na resposta do paciente no período pós-operatório. Rev Esc Enferm USP 1999;33(4):391-403.

15. Lima FET, Araujo TL, Serafim ECG, Custodio IL. Nursing consultation protocol for patients after myocardial revascularization: influence on anxiety and depression. Rev Latinoam Enferm 2010;18(3):331-8.

16. Marcolino JAM, Mathias LAST, Piccinini Filho L, Guaratini AA, Suzuki FM, Alli LAC. Escala hospitalar de ansiedade e depressão: estudo da validade de critério e da confiabilidade com pacientes no pré-operatório. Revista Brasileira de Anestesiologia 2007;57(1):52-62.

17. Botega NJ, Bio MR, Zomignani MA, Garcia Junior C, Pereira WAB. Transtornos de humor em enfermarias de clínica médica e validação de escala de medida (HAD) de ansiedade e depressão. Rev Saúde Pública 1995;29:355-63.

18. Medeiros VCC, Peniche ACG. A influência da ansiedade nas estratégias de enfrentamento utilizadas no período pré-operatório. Rev Esc Enferm USP 2006;40(1):86-92.

19. Peniche ACG, Chaves EC. Algumas consideraçôes sobre o paciente cirúrgico e a ansiedade. Rev Latinoam Enferm 2000;8(1):45-50.

20. Bachion MM, Magalhães FGS, Munari DB, Almeida SP, Lima ML. Identificação do medo no período pré-operatório de cirurgia cardíaca. Acta Paul Enferm 2004;17(3):298-304.

21. Vargas TVP, Maia EM, Dantas RAS. Patient feelings during the preoperative period for cardiac surgery. Rev Latinoam Enferm 2006;14(3):383-8.

22. Andrade LM, Soares E. Refletindo sobre a ansiedade do paciente no período pós-operatório de cirurgia cardíaca de emergência: um estudo de caso. Rev RENE 2001;2(1):86-9.

23. Grazziano ES, Bianchi ERF. Nível de ansiedade de clientes submetidos a cineangiocoronariografia e de seus acompanhantes. Rev Latinoam Enferm 2004;12(2):168-74.

24. Boschco M, Mantovani M. As percepçóes dos portadores de insuficiência cardíaca frente ao seu processo de adoecimento. Cienc Cuid Saúde 2007;6(4):463-70.

25. Ferrari CMM, Sousa RMC, Garzon E. Orientaçôes ao paciente portador de epilepsia submetido ao vídeo-EEG: comparação dos níveis de ansiedade com o uso de diferentes estratégias. Arq Neuro-Psiquiatr 2005;63(4):1028-34.

26. Haddad MCL, Zago E, Andreassa FJ. Desconfortos referidos por indivíduos submetidos à ressonância magnética. Ciênc Cuid Saúde 2005;4(2):149-55.

27. Frias TFP, Costa CMA, Sampaio CEP. O impacto da visita pré-operatória de enfermagem no nivel de ansiedade de pacientes cirúrgicos. Rev Min Enferm 2010;14(3):345-52.

28. Ribeiro V, Munari DB. Saúde mental em clientes cirúrgicos: o desenvolvimento de açôes de enfermagem através do grupo de suporte/apoio. Rev Bras Enferm 1998;51(1):147-64.

29. Gomes VM, Silva MJP, Araújo EAC. Efeitos gradativos do toque terapêutico na reduçáo da ansiedade de estudantes universitários. Rev Bras Enferm 2008;61(6):841-6. 